\title{
Aplicação do mapeamento conceitual na regulação do acesso aos serviços públicos de saúde, Distrito Federal, Brasil
}

\author{
Application of conceptual mapping in the regulation of access \\ to public health services in the Federal District, Brazil
}

Geisa Cristina Modesto Vilarins (https://orcid.org/0000-0002-8341-7420) ${ }^{1}$

Diana Lúcia Moura Pinho (https://orcid.org/0000-0003-4212-2340) ${ }^{2}$

\footnotetext{
${ }^{1}$ Programa de PósGraduação em Ciências e Tecnologias em Saúde, Universidade de Brasília (UnB). Campus Universitário Darcy Ribeiro s/n, Asa Norte. 70910900 Brasília DF Brasil. ggvilarins@gmail.com ${ }^{2}$ Departamento de Enfermagem, Faculdade de Ciências da Saúde, UnB. Brasília DF Brasil.
}

\begin{abstract}
Health regulation in the pursuit of equity is the goal of management and requires evaluation methods that improve work processes. The scope of this article is to analyze the application of conceptual mapping in the regulation of access to public health services. It is an exploratory and descriptive study, using a mixed approach, carried out at the Health Regulatory Complex of the Federal District. The data were collected between August and October 2019 and analyzed with the assistance of IRaMuTeQ and Concept Systems ${ }^{\circledR}$ software. There is a convergence of the 25 statements generated by the 71 participants, grouped into 4 clusters, on regulation towards the principle of equity. The healthcare priority level was presented as the focus of regulation and management, the driving force behind the integration of processes. Conceptual mapping is a tool that can support regulation planning and evaluation, as it makes it possible to identify priority points to be worked on by management in improving the regulatory processes identified in this study. These include the training of professionals, the transparency of information and the level of priority healthcare, for effective, equitable, rational and timely access for users of the health system.

Key words Healthcare regulation and monitoring, Accessibility of health services, Conceptual mapping, Health management, Health evaluation
\end{abstract}

Resumo A regulação em saúde na busca da equidade subsidia a gestão e necessita de métodos avaliativos que incrementem os processos de trabalho. O objetivo deste artigo é analisar a aplicação do mapeamento conceitual na regulação do acesso aos serviços públicos de saúde. Estudo exploratório e descritivo, sob abordagem mista, realizado no Complexo Regulador em Saúde do Distrito Federal. Os dados foram coletados entre agosto e outubro de 2019 e analisados com o auxílio dos softwares IRaMuTeQ e Concept Systems ${ }^{\circledR}$. Há uma convergência de 25 declarações geradas pelos 71 participantes, agrupadas em 04 Clusters sobre a regulação rumo ao princípio da equidade. O nível de prioridade assistencial apresentou-se como o foco da regulação e a gestão, a força motriz para a integração dos processos. O mapeamento conceitual é uma ferramenta que pode apoiar o planejamento e avaliação da regulação, pois possibilita identificar pontos prioritários a serem trabalhados pela gestão na melhoria dos processos regulatórios evidenciados neste estudo como a capacitação dos profissionais, a transparência da informação e o nível de prioridade assistencial para um acesso efetivo, equânime, racional e oportuno aos usuários do sistema de saúde.

Palavras-chave Regulação e fiscalização em saúde, Acesso aos serviços de saúde, Mapeamento conceitual, Gestão em saúde, Avaliação em saúde 


\section{Introdução}

O acesso aos serviços de saúde tem sido alvo de debates em todo o mundo há várias décadas, visto que envolve não só a relação entre oferta e demanda, mas uma rede de interações e contextos multidimensionais e dinâmicos ${ }^{1-3}$. Tais discussões perpassam políticas públicas de saúde, políticas sociais e econômicas fundamentais para o desenvolvimento de planos e metas sustentáveis no setor saúde, considerando os cenários nacionais e internacionais ${ }^{3}$.

Como objeto explícito de muitos sistemas de saúde, a busca por equidade no acesso aos serviços se depara com inúmeras barreiras sejam físicas, financeiras e outras para a utilização dos mesmos em tempo oportuno, de modo a atender às necessidades do usuário ${ }^{4-6}$. Estreita-se, assim, a relação do conceito de regulação ao princípio da equidade, demonstrando que a regulação é uma importante ferramenta para a efetivação desse princípio $^{7-9}$, controlando não só o cumprimento das leis para a proteção dos indivíduos e das comunidades vulneráveis, como também controla a qualidade dos serviços ${ }^{10}$.

Trata-se de um processo pelo qual a atividade do setor público e as forças de mercado são direcionadas para o bem da população ${ }^{11}$, fazendo com que os gestores, na busca da equidade do acesso aos serviços de saúde ${ }^{12}$, utilizem a regulação como instrumento de gestão tornando-a um potente equalizador social do sistema ${ }^{13}$.

No Brasil, sob a égide constitucional, a Política Nacional de Regulação ${ }^{14}$ do Sistema Único de Saúde (SUS) objetiva, precipuamente, promover a equidade do acesso à saúde, considerando a regulação como um instrumento de gestão que viabiliza o ordenamento da relação dos usuários com os prestadores de saúde, além de apresentar uma estreita interface com o planejamento, o controle, a avaliação e com os diversos níveis de complexidade da assistência, buscando garantir a assistência integral aos usuários do SUS ${ }^{15,16}$.

Tal política norteou a criação de Complexos Reguladores e Centrais de Regulação em todo o país com o intuito de intermediarem a demanda dos usuários por serviços de saúde ${ }^{17}$. Para este estudo, o Complexo Regulador em Saúde do Distrito Federal foi escolhido como campo empírico de pesquisa por apresentar um conjunto de estratégias que visam o acesso por meio da organização de uma rede pública articulada hierarquicamente, tendo como um dos seus desafios a adoção de mecanismos de incorporação tecnológica para consolidar as ações desenvolvidas por todas as unidades que o compõe ${ }^{18}$.
Por ser a regulação do acesso uma política de saúde e se apresentar como um importante papel frente ao planejamento estratégico, controle e monitoramento das ações e a avaliação tecnológica em saúde ${ }^{19}$, questionou-se, neste estudo, a possibilidade do uso do mapeamento conceitual como um instrumento metodológico viável para analisar os processos de regulação do acesso aos serviços de saúde.

O mapeamento conceitual é definido como um método híbrido que utiliza abordagens qualitativas e quantitativas, permitindo que ideias ou conceitos sejam representados em um dispositivo gráfico hierárquico ${ }^{20}$. Tem aplicação em diversas áreas do conhecimento como educação, biologia e também em serviços de gerenciamento, inteligência artificial, telemedicina, entre outros, desde níveis informais até níveis formais, como em sistemas especializados ${ }^{21-23}$, além de ser utilizado como instrumento de análise no campo da equidade no acesso aos cuidados de saúde ${ }^{20}$.

Trochim ${ }^{20}$, idealizador do mapeamento conceitual, descreve seu uso em variados contextos e complexidades desde ações básicas de saúde, planejamento, tomada de decisão, avaliação, desenvolvimento de diretrizes para a gestão pública em sistemas de saúde, tornando-se uma ferramenta viável e importante para processos que tenham rigor e credibilidade científica ${ }^{24-27}$. Da mesma forma, os estudos de Donnelly ${ }^{28}$ realizados em 35 universidades dos Estados Unidos e Canadá, no período compreendido entre 1985 e 2014, aplicaram o mapeamento conceitual em diferentes áreas do conhecimento, incluindo planejamento e avaliação na área da saúde. Já para Andersen e Slonim $^{29}$, o mapeamento conceitual é útil para subsidiar não só o planejamento e a avaliação, mas, principalmente, instrumentalizar as definições de prioridades em saúde pública.

Em se tratando de políticas de saúde e planos de desenvolvimento para o acesso da população, os estudos de Goicolea et al..$^{30}$ na Suécia, Bradbury-Jones et al. ${ }^{31}$ na Escócia, Johnson et al. ${ }^{32}$ e Willging et al. ${ }^{33}$ nos Estados Unidos contribuíram, mediante o uso do mapeamento conceitual, para a construção de novas diretrizes e debates na reformulação das políticas vigentes, o que fortalece o discurso da ampliação do acesso aos serviços de saúde, corroborado pelos estudos de Ahmad et al. ${ }^{34} \mathrm{e}$ Vives-Cases et al. ${ }^{35}$ que identificaram as principais barreiras que impedem tal acesso.

Vale ressaltar a diferença metodológica entre mapeamento conceitual e mapa conceitual ou cognitivo apesar de serem usados como sinônimos. Este último foi idealizado por Novak e 
Gowin $^{36}$ na área de educação como uma ferramenta com potencial para favorecer a aprendizagem significativa, tendo aplicação em diversas áreas do conhecimento. $\mathrm{O}$ mapa conceitual não é um método híbrido como o mapeamento conceitual, mas considera a necessidade de conhecer as ideias e conceitos prévios e a estrutura dos significados com o propósito de estabelecer conhecimentos inter-relacionados e hierarquizados por uma frase de ligação, em um dispositivo gráfico, gerando uma proposição ${ }^{37}$. O estudo de Lins et al. ${ }^{38}$, por exemplo, relata a medição de indicadores de desempenho da assistência à saúde em 5.565 municípios brasileiros, por meio do mapa conceitual para apoiar a formulação de políticas de saúde.

Apesar da aplicabilidade do mapeamento conceitual ocorrer em diversas áreas do conhecimento ${ }^{30,31,34,35}$, ainda há poucos estudos publicados no Brasil que relatam o uso do mapeamento conceitual na área da saúde especificamente, assim como uma escassez de pesquisas que versem sobre métodos que analisem processos de regulação de serviços de saúde. O estudo de Figueiredo e $\mathrm{Hartz}^{39}$ traz uma reflexão sobre qual método deve ser adotado para avaliar a regulação em saúde, considerando fatores políticos e sociais, para além dos técnicos e mercadológicos, não se encaixando em modelos de avaliação já pré-definidos.

Portanto, pretende-se com este trabalho analisar a aplicação do mapeamento conceitual na regulação do acesso aos serviços públicos de saúde com vistas à subsidiar os processos de planejamento, avaliação, controle, monitoramento e tomada de decisão inerentes à gestão dos sistemas de saúde.

\section{Material e métodos}

Trata-se de um estudo exploratório e descritivo em uma abordagem de método misto, realizado no Complexo Regulador em Saúde do Distrito Federal. A pesquisa atendeu às normas éticas fundamentadas na Resolução CNS/MS 466/2012 a aprovada pelo Comitê de Ética em Pesquisa da Fundação de Ensino e Pesquisa em Ciências da Saúde.

Ao todo, foram identificados 579 servidores atuantes na área de regulação do acesso aos serviços públicos de saúde do DF, sendo 24 gestores e 555 demais profissionais. Como critério de inclusão, foram eleitos 96 participantes após responderem completamente um survey on line referente a uma outra parte desta pesquisa, sendo que destes, 20 eram gestores e 76 demais profissionais.

O instrumento de coleta de dados referente a este estudo foi enviado aos 96 participantes, finalizando a amostra em 71 respondentes que completaram todas as fases. O cálculo amostral foi atendido considerando o grau de confiança de $90 \%$ e margem de erro de $5 \%$ para significância estatística ${ }^{40}$. Os dados foram coletados no período de agosto a outubro de 2019 em duas fases, seguidos do processamento e a análise dos mesmos por programas informáticos específicos.

Para fins de pesquisa, este estudo adotou o referencial teórico de Trochim ${ }^{20,24-27}$ que recomenda a elaboração do mapeamento conceitual em seis etapas, processadas e analisadas pelo Concept Systems ${ }^{\circledR 27}$, são elas:

1. Brainstorming - Consistiu na elaboração do Focus Prompt (pergunta foco) "A regulação do acesso aos serviços públicos de saúde é...”, funcionando como um disparador rápido para que os 96 participantes da pesquisa completassem a frase com ideias, conceitos, características, pontos fortes e desafios sobre o tema, por meio de survey on line. Esta foi a opção escolhida para a captação dos dados, visto a limitação do Concept Systems ${ }^{\circledR}$ que ainda não possui versão em língua portuguesa. Neste sentido, o Focus Prompt foi transformado em um instrumento de coleta de dados com duas questões abertas: "O que você entende como regulação do acesso aos serviços públicos de saúde?" e "Indique pontos fortes e desafios que poderiam melhorar os processos de trabalho na sua área de atuação na regulação”. Isto permitiu obter maior profundidade e especificidade nas respostas, sendo o conteúdo destas submetido ao software livre IRaMuTeQ (Interface de $R$ pour les Analyses Multidimensionnelles de Textes et de Questionnaires) com o intuito de produzir a Classificação Hierárquica Descendente (CHD) e a Nuvem de Palavras que geraram classes dos segmentos de textos com vocabulário semelhante entre si e diferente dos segmentos de texto das outras classes, demonstrando as relações existentes entre elas ${ }^{41-43}$. Esta fase foi concluída com 71 respondentes, sendo 20 gestores e 51 demais profissionais, habilitando-os a prosseguirem às fases seguintes.

2. Ordenação dos Dados (Sorting) - Esta foi uma etapa de organização das ideias coletadas no Brainstorming. As classes geradas pelo IRaMuTeQ, juntamente com frequência das palavras em cada classe e a Nuvem de Palavras possibilitaram a elaboração de 25 Statements (declarações) 
conceitos) como resultantes das respostas dadas pelos participantes quanto ao Focus Prompt. Estes Statements foram disparados novamente, aos mesmos 96 participantes, via e-mail, por meio de um survey on line, constituindo a segunda etapa deste estudo, possibilitando que fossem avaliados quanto à classificação de importância.

3. Classificação e Análise dos Dados (Rating) Nesta fase, os participantes da pesquisa classificaram os 25 Statements por ordem de importância ou prioridade. Para otimizar a classificação, foi utilizada a Escala Likert de 6 pontos, na qual o valor 1 corresponde ao conceito "extremamente importante" e o valor 6 corresponde ao conceito "pouco importante".

4. Análise de Clusters - Após a classificação dos Statements realizou-se a construção da interface do Mapa de Pontos, no Concept System. Nesta etapa, cada Statement foi considerado um ponto que se relaciona com outro gerando uma relação de significados. Para isso, o sistema gerou na análise dos dados, um ponto de corte de similaridade o qual filtrou as relações entre os Statements considerados ilegítimos. Ao executar a análise, o sistema forneceu o valor de stress (tensão) o qual representa as interações entre o número de filtros empregados e o ponto de corte de similaridade. Por fim, foi gerado um mapa de Clusters representando áreas e temas diferentes que podem ter uma convergência conceitual.

5. Interpretação dos Mapas - nesta fase a estrutura conceitual e o resultado básico do processo de mapeamento foram constituídos apontando os possíveis caminhos para as soluções do problema de pesquisa. A estrutura apresentada possibilitou a compreensão das questões principais as quais são representadas por pontos que foram considerados na análise do processo de regulação do Distrito Federal.

6. Aplicação do Mapa Conceitual - nesta etapa foi realizado o confronto analítico entre o que foi apontado na geração dos mapas e a motivação da realização do processo. Forneceu assim, a fundamentação (conceitual)e os esforços exigidos na busca das melhorias para a avaliação das ações em curso e o seu planejamento, colocando em evidência os pontos que devem ser priorizados e a relação entre eles.

\section{Resultados}

Em relação aos resultados obtidos com o auxílio do software IRaMuTeQ, a CHD final de acordo com a análise do conteúdo das questões abertas adaptadas do Focus Prompt gerou 92 Unidades de
Contexto Inicial (UCI) que representa o Corpus textual. Destas, foram derivadas 226 Unidades de Contexto Elementar (UCE) que são os segmentos de texto com vocabulário semelhante entre si e diferentes das UCE das outras classes. E destas foram classificados 162 segmentos de texto, subdivididos em 05 classes, perfazendo um total de $71,68 \%$ de aproveitamento, demonstrando o agrupamento das palavras estatisticamente significativas ${ }^{40,43,44}$.

No Dendrograma apresentado na Figura 1, o Corpus de texto foi dividido em dois Subcorpus. No primeiro, obteve-se a Classe 5 com 38 UCE, que correspondeu a $23,5 \%$ do total. Neste mesmo subcorpus houve uma segunda subdivisão, que englobou a Classe 3 com 37 UCE e a Classe 1 com 24 UCE, sendo 22,8\% referente à Classe 3 e $14,8 \%$ à Classe 1 do total das UCE. Do outro subcorpus, obteve-se a Classe 2, com 24 UCE, que correspondeu a 16,7\% das UCE, e a Classe 4 , constituída de 36 UCE, e que concentrou 22,2\% das UCE do Corpus total.

Para cada classe foi computada uma lista de palavras com maior frequência a partir do teste $\chi^{2}$. Após a leitura minuciosa e exaustiva de todas as palavras que compõem as classes, estas foram nominadas de modo a identificá-las quanto à representatividade do tema central deste estudo. Assim, cada classe passou a se chamar: Classe 1 - Conceitos de regulação; Classe 2 - Desafios da regulação; Classe 3 - Dificuldades da regulação; Classe 4 - Processo regulatório; Classe 5 - Objetivos da regulação.

As classes geradas demonstraram as palavras com maior significância estatística por apresentarem $\chi^{2} \geq 3,84$ como indicador da associação da palavra à classe, com uma margem de erro $\leq 0,05$ e $\mathrm{p}<0,0001$, conforme a Tabela 1 , garantindo a validade de cada classe apresentada.

Outra forma visual que apresenta os principais pontos de convergência citados pelos participantes quanto à descrição de suas percepções acerca da regulação do acesso aos serviços públicos de saúde foi a Nuvem de Palavras (Figura 2) gerada com o auxílio do software IRaMuTeQ. A Nuvem de Palavras agrupa e organiza as palavras graficamente em função da sua frequência. As palavras mais frequentes encontradas foram "regulação", "serviço", “saúde”, “processo", “acesso", “sistema”, “demanda”, “oferta”, "leito”, “rede”, "necessidade", "recurso", "equidade”, entre outras.

Neste sentido, este agrupamento e a organização das palavras por frequência ratificou a CHD possibilitando avançar rumo à formulação dos conceitos (Statements) necessários ao sequenciamento do estudo. 


\begin{tabular}{|c|c|c|c|c|}
\hline classe 2 & classe 4 & classe 3 & classe 1 & classe 5 \\
\hline $16.7 \%$ & $22.2 \%$ & $22.8 \%$ & $14.8 \%$ & $23.5 \%$ \\
\hline $\begin{array}{l}\text { momento } \\
\text { regulador } \\
\text { treinamento } \\
\text { entender } \\
\text { equipa } \\
\text { maior servidor } \\
\text { como } \\
\text { mais } \\
\text { capacitação } \\
\text { atendimento } \\
\text { médico } \\
\text { plantão } \\
\text { necessidade } \\
\text { oportuno } \\
\text { complexo } \\
\text { solicitação } \\
\text { motivar } \\
\text { externo } \\
\text { centro } \\
\text { ao } \\
\text { técnica } \\
\text { vez } \\
\text { mesmo } \\
\text { precisar } \\
\text { implantação }\end{array}$ & $\begin{array}{l}\text { leito } \\
\text { uti } \\
\text { unidade } \\
\text { paciente } \\
\text { enfermaria } \\
\text { criação } \\
\text { perfil } \\
\text { utis } \\
\text { integrar } \\
\text { suporte } \\
\text { acordo } \\
\text { referência } \\
\text { transporte } \\
\text { utilização } \\
\text { hospital } \\
\text { regular } \\
\text { vaga } \\
\text { não } \\
\text { rede } \\
\text { região } \\
\text { cuidado } \\
\text { considerar } \\
\text { manutenção } \\
\text { monitorar } \\
\text { etc } \\
\text { dar } \\
\text { alto samu }\end{array}$ & $\begin{array}{l}\text { capaz } \\
\text { regulação } \\
\text { nível } \\
\text { escassez } \\
\text { prioridade } \\
\text { assitencial } \\
\text { permitir } \\
\text { grande } \\
\text { princípio } \\
\text { melhoria } \\
\text { diretor } \\
\text { integração } \\
\text { existente } \\
\text { demanda } \\
\text { recurso } \\
\text { central } \\
\text { quando } \\
\text { necessitar } \\
\text { espaço } \\
\text { construção } \\
\text { gestão } \\
\text { clínico } \\
\text { dado } \\
\text { sus } \\
\text { análise }\end{array}$ & $\begin{array}{l}\text { serviço } \\
\text { equidade } \\
\text { oferecer } \\
\text { saúde } \\
\text { acesso } \\
\text { ferramenta } \\
\text { integralidade } \\
\text { ordenar } \\
\text { otimizar } \\
\text { forma } \\
\text { qualificar } \\
\text { população } \\
\text { melhor } \\
\text { transparência } \\
\text { necessidade } \\
\text { usuário } \\
\text { equânime } \\
\text { ação } \\
\text { garantir } \\
\text { também } \\
\text { justo } \\
\text { objectivo } \\
\text { integral } \\
\text { primário }\end{array}$ & $\begin{array}{l}\text { órgão } \\
\text { consulta } \\
\text { procedimento } \\
\text { esperar } \\
\text { transplante } \\
\text { listar } \\
\text { gerenciar } \\
\text { fila } \\
\text { aumento } \\
\text { proporcionar } \\
\text { df } \\
\text { controlo } \\
\text { monitoramento } \\
\text { doação } \\
\text { melhorar } \\
\text { qualificação } \\
\text { além } \\
\text { informação } \\
\text { exame } \\
\text { gerar } \\
\text { número } \\
\text { transparência } \\
\text { marcação } \\
\text { papel } \\
\text { estabelecer } \\
\text { atividade }\end{array}$ \\
\hline
\end{tabular}

Figura 1. Dendrograma por classes e palavras com maior qui-quadrado $(\chi 2)$ fornecido pelo software IRaMuTeQ, Brasília, Brasil, 2019.

Legenda: Classe 1 - Conceitos de Regulação; Classe 2 - Desafios da Regulação; Classe 3 - Dificuldades da Regulação; Classe 4 Processo Regulatório e Classe 5 - Objetivos da Regulação.

Fonte: Elaborado pelas autoras.

Tabela 1. Classificação Hierárquica Descendente sobre a Regulação do acesso aos serviços públicos de saúde no Distrito Federal, Brasil, 2019.

\begin{tabular}{|c|c|c|c|}
\hline Classe & Identificação & UCE (\%) & Palavras associadas ( $2 \geq 3,84 \mathrm{e} \mathrm{p}<0,0001)$ \\
\hline 1 & $\begin{array}{l}\text { Conceitos de } \\
\text { Regulação }\end{array}$ & $24(14,8)$ & $\begin{array}{l}\text { serviço }(27,6) \text {, equidade }(26,7) \text {, oferecer }(23,5) \text {, saúde }(21,2) \text {, } \\
\text { acesso }(21,1) \text {, ferramenta }(20,3) \text {, integralidade }(17,5) \text {, ordenar } \\
(17,5) \text {, otimizar }(17,3) \text {, forma }(15,6) \text {, qualificar }(15,1) \text {. }\end{array}$ \\
\hline 2 & Desafios da Regulação & $27(16,7)$ & $\begin{array}{l}\text { momento }(31,1) \text {, regulador }(26,7) \text {, treinamento }(20,5) \text {, entender } \\
(20,5) \text {, equipa }(20,3) \text {, maior }(20,0) \text {, servidor }(16,2) \text {. }\end{array}$ \\
\hline 3 & $\begin{array}{l}\text { Dificuldades da } \\
\text { Regulação }\end{array}$ & $37(22,8)$ & $\begin{array}{l}\text { capaz }(21,0) \text {, regulação }(19,4) \text {, nível }(16,6) \text {, escassez }(13,8) \text {, } \\
\text { prioridade }(11,5) .\end{array}$ \\
\hline 4 & Processo regulatório & $36(22,2)$ & $\begin{array}{l}\text { leito }(70,3) \text {, uti }(53,6) \text {, unidade }(25,7) \text {, paciente }(18,4) \text {, } \\
\text { enfermaria }(17,0) \text {, criação }(17,0) \text {, perfil }(17,0) \text {. }\end{array}$ \\
\hline 5 & $\begin{array}{l}\text { Objetivos da } \\
\text { Regulação }\end{array}$ & $38(23,5)$ & $\begin{array}{l}\text { órgão }(33,5) \text {, consulta }(31,1) \text {, procedimento }(31,1) \text {, esperar } \\
(27,46) \text {, transplante }(22,3) \text {, listar }(16,8) \text {, gerenciar }(16,8) \text {, fila } \\
(15,7) \text {. }\end{array}$ \\
\hline
\end{tabular}

Fonte: Elaborado pelas autoras. 


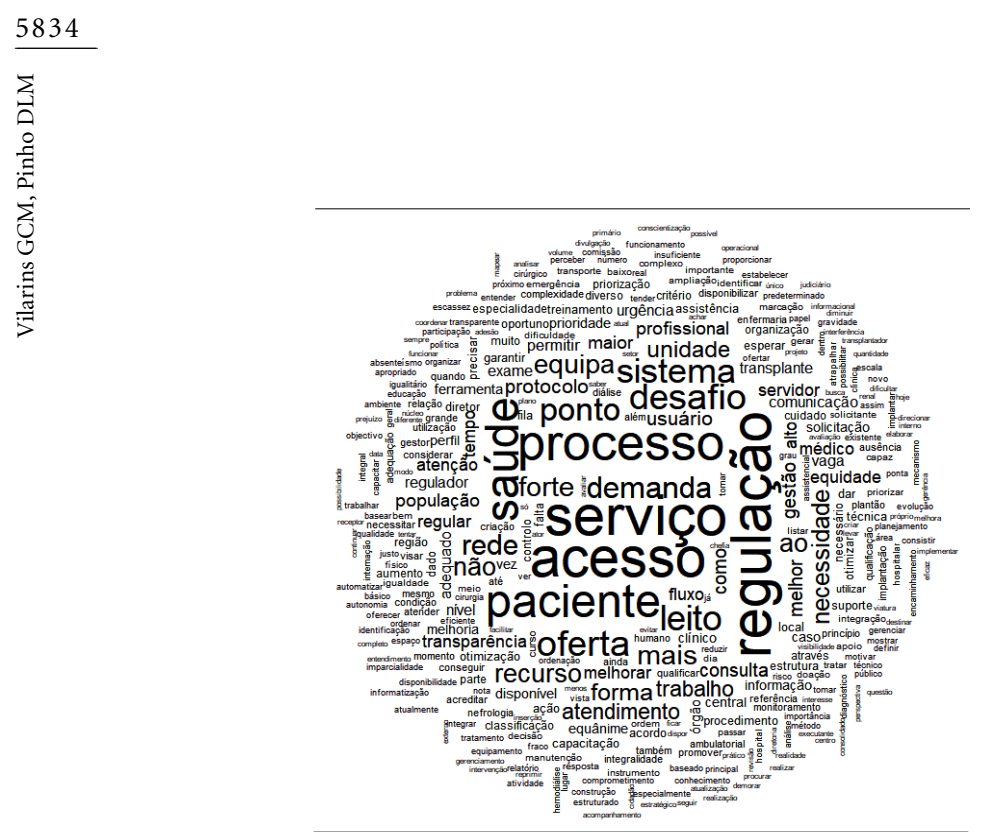

Figura 2. Nuvem de palavras obtida pelo software IRaMuTeQ, Brasília, Brasil, 2019.

Fonte: Elaborado pelas autoras.

O processamento dos dados, com o auxílio do software Concept Systems ${ }^{\circledR}$, gerou inicialmente o Mapa de Pontos de acordo com a análise multivariada, a partir da matriz de similaridade resultante do agrupamento realizado nas etapas de Sorting e Rating, representando o conjunto de Statements gerados pelos participantes. Nesse mapa, cada um dos Statements foi plotado em um plano XY, com seus respectivos números de identificação. Os 25 Statements classificados pelos participantes estão representados na Figura 3, na forma de um Mapa de Pontos, são eles:

1. É um serviço que oferece equidade.

2. É uma ferramenta que ordena e otimiza o acesso à saúde para a população.

3. É uma ação que garante melhor transparência aos usuários.

4. É uma forma de qualificar a necessidade da população.

5. É uma ferramenta de acesso para garantir a integralidade.

6. Deve considerar o treinamento das equipes.

7. Deve considerar a capacitação do regulador/médico.

8. Deve entender o momento oportuno do atendimento à solicitação/necessidade.

9. Deve motivar o servidor.

10. Deve ser capaz de melhorar a integração com as centrais.
11. Deve ser capaz de melhorar a escassez de recursos.

12. Deve ser capaz de melhorar a gestão/direção.

13. Deve ser capaz de melhorar o nível de prioridade assistencial.

14. Deve ser capaz de melhorar a demanda existente.

15. Deve ser capaz de melhorar o espaço/ construção das centrais.

16. Deve considerar o suporte do leito/unidade/UTI/enfermaria/hospital.

17. Deve considerar o perfil do paciente.

18. Deve considerar a referência da região.

19. Deve considerar o nível de prioridade assistencial.

20. Deve considerar a vaga na rede.

21. Deve considerar o transporte.

22. É gerenciar a fila de espera (consulta/exame/procedimento/transplante de órgãos).

23. É gerar transparência da informação.

24. É proporcionar controle e monitoramento

25. É melhorar a qualificação da informação.

Os pontos muito próximos significam uma média de classificação mais frequente do que outros pontos que se mostram distantes.

O valor de stress do Mapa de Pontos indica que, quanto menor o valor de stress encontrado, menos ponto de corte de similaridade é empregado, o que significa condições ideais e suficientes para a interpretabilidade dos dados. $\mathrm{O}$ valor de stress varia de 0.1 a 0.35 e o mapa resultante dentro desta faixa é interpretado como indicador para o prosseguimento da análise. Neste estudo o valor de stress encontrado foi de 0.2422 frente às 12 interações resultantes da classificação dos 25 Statements.

A análise hierárquica de Clusters foi o segundo processamento conduzido para representar o domínio conceitual no mapeamento no qual os Statements formaram o Mapa de Clusters que reflete conceitos similares, conforme apresentado na Figura 4, resultando no Mapeamento Conceitual obtido como resultado do presente estudo. Os Clusters representam a proximidade do tema dos discursos dos participantes, pois quanto mais próximos, mais parecido é o assunto tratado.

Cada cluster foi nominado de acordo com a predominância dos Statements em cada um de modo que a identificá-lo pelo tema que mais o representa. Assim, o Cluster 1 foi identificado como Conceitos de Regulação; Cluster 2 - Finalidades da Regulação; Cluster 3 - Processo Regulatório e Cluster 4 - Desafios da Regulação. 


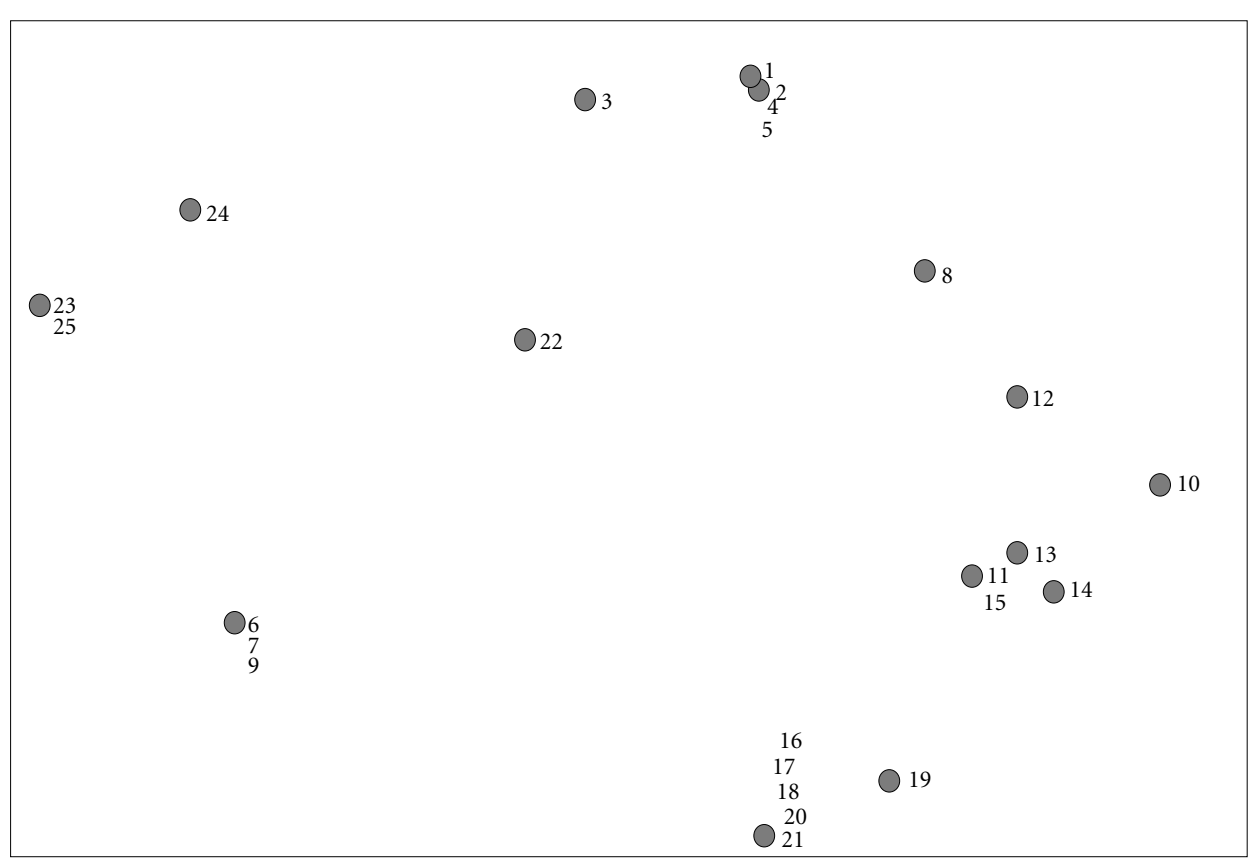

Figura 3. Mapa de Pontos gerado pelo software Concept Systems ${ }^{\circledR}$ representando a percepção conceitual sobre a regulação do acesso aos serviços públicos de saúde no Distrito Federal, Brasil, 2019.

Fonte: Elaborado pelas autoras.

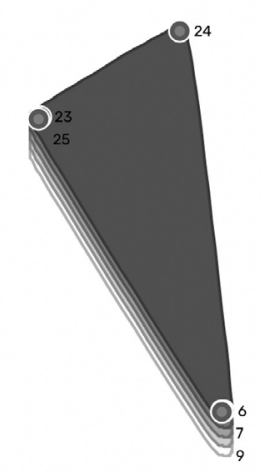

:: 1 Finalidades da Regulação

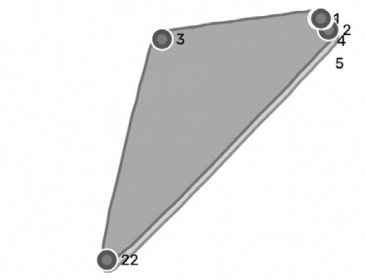

::2 Conceitos de Regulação

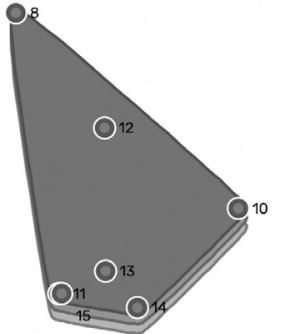

:4 Desafios da Regulação 
Os valores de ponte identificados em cada Cluster refletem a ancoragem dos Statements e a relação entre eles variou de 0 a 1 . O Cluster 1 apresentou um valor de ponte 0,33, Cluster 2 0,83; Cluster 3-0,07 e Cluster 4-0,37. Quanto menor o valor, mais próxima a relação entre os Statements colocando em evidência que as ideias ou conceitos citados pelos participantes se aproximam de forma significativa. Observa-se ainda, que quanto maior número de camadas contidas em um cluster, mais alto será o valor de ponte agregando ainda mais as ideias.

Em relação ao grau de importância de cada Cluster, quanto maior foi o número de camadas ou layers, mais importante é o grupo e os valores são representados pela altura média de suas camadas. Assim, o grau de importância do Cluster 1 foi de 2,81; Cluster 2 - 2,69; Cluster 3 - 3,50 e Cluster 4-3,29.

\section{Discussão}

O mapeamento conceitual deve ser visto como diagrama hierárquico que indica relações entre conceitos os quais procuram refletir a organização conceitual de um contexto ou parte dele. São diagramas de significados, de relações significativas entre definições; de hierarquias conceituais. Eles permitem classificar conceitos, relacioná-los e hierarquizá-los ${ }^{24,45}$. Sob esta lógica, as análises realizadas resultaram em ferramentas gráficas para a organização e representação do conhecimento $^{27}$.

O uso dos softwares IRaMuTeQ e Concept Systems permitiu compreender o conteúdo das respostas a partir do Focus Prompt na tentativa de identificar as inferências mais plausíveis, a partir dos dados coletados, não sendo, portanto, um processo automático, mas uma etapa analítica em que o pesquisador é o elemento central ${ }^{46}$.

Considerando a etapa de Brainstorming, a divisão observada na CHD indica a Classe 5 (Objetivos da Regulação) como a mais representativa com $23,5 \%$ de aproveitamento do total das UCE, transparecendo as ideias sobre os pontos fortes que a regulação do acesso aos serviços públicos de saúde apresenta, segundo os participantes:

[...] a regulação do acesso aos serviços de saúde se sustenta na construção de estruturas organizacionais capazes de concretizar na prática os princípios e diretrizes do SUS coordenando as demandas com as ofertas locais de forma eficiente e equânime em qualquer contexto desde a suficiência até a es-

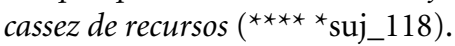

Há uma relação direta entre as Classes 2 (Desafios da Regulação) e 4 (Processo Regulatório) por possuírem discursos bem próximos, pois aparecem dentro da mesma subclasse. Subentende-se, neste sentido, que ao se pensar em processo regulatório também é necessário identificar os desafios existentes e as habilidades para superá-los.

Da mesma forma, as Classes 1 (Conceitos de Regulação) e 3 (Dificuldades da Regulação) apresentam-se na mesma subclasse remetendo às ideias de que as definições de regulação esbarram em dificuldades para execução das ações pertinentes ao processo regulatório:

[...] consiste em um mecanismo de ordenação e organização que tenta promover o acesso de forma equânime, adequada e oportuna por meio da utilização de protocolos clínicos, especialmente em nosso meio onde há desequilíbrio entre a demanda e a oferta (**** suj_95).

Em relação à Nuvem de Palavras (Figura 2), nota-se uma convergência da percepção dos participantes em seus discursos sobre conceitos de regulação, aludindo ao exposto por Lima ${ }^{9} q u e$ define a regulação como um serviço que utiliza mecanismos para facilitar o acesso ao sistema de saúde, com base nos princípios da equidade e da integralidade e na adequação da oferta dos serviços de atenção à saúde com relação às necessidades da população:

[...] ferramenta de gestão que viabiliza o acesso de cidadãos aos serviços de saúde de forma oportuna adequada, equânime, baseada em critérios clínicos e de priorização conforme as necessidades do paciente e suporte oferecido pelas unidades componentes das redes de atenção em suas mais variadas instâncias primárias, secundária, terciária (**** ${ }^{*}$ suj_88).

Sobre o Mapa de Clusters (Figura 4) produzido pelo software Concept Systems ${ }^{\circledR}$ e segundo a análise de abrangência, a qual é traduzida pelas ligações que refletem as aproximações das classificações das ideias ${ }^{27}$, é possível inferir que os Clusters 1 e 4 apresentam distâncias próximas se comparados à distância entre os Clusters $2 \mathrm{e}$ 3 , demonstrados pelos valores de ponte encontrados. Isto quer dizer que os clusters que estão próximos são mais similares conceitualmente do que os clusters mais afastados. De fato, ideias que remetem a conceitos e definições de regulação (Cluster 1) estão mais relacionadas com os desafios (Cluster 4) a serem enfrentados. Por outro lado, há um distanciamento do que se tem como processo regulatório per si (Cluster 3) e as finalidades da regulação (Cluster 2). A distância não é 
o reflexo apenas da forma como foram classificados os Statements, mas como cada ponto tem seu próprio conjunto de relacionamentos, de modo que cubra a ideia básica a que se relaciona.

Considerando o Cluster 1 - Conceitos de Regulação, observa-se que as ideias propostas nos Statements 1, 2, 4 e 5 estão distantes dos Statements 3 e 22, apesar de pertencerem ao mesmo cluster. Pela percepção dos participantes, a regulação do acesso aos serviços de saúde pode ser entendida como um serviço ou ferramenta que oferece equidade, garante a integralidade, ordena e otimiza o acesso à saúde com base na qualificação das necessidades da população, convergindo, assim, ao preconizado pela Política Nacional de Regulação ${ }^{14}$.

No entanto, a regulação do acesso ainda é uma ação que precisa garantir melhor a transparência aos usuários em relação ao gerenciamento da fila de espera seja por uma consulta, exame, procedimento, internação em leito geral/UTI ou até mesmo para um transplante de tecidos e órgãos para que se tenha a devida e equânime ampliação do acesso:

[...] consiste na ordenação e qualificação dos fluxos de acesso às ações e serviços de saúde de modo a otimizar a utilização dos recursos assistenciais disponíveis e promover a transparência a integralidade e a equidade no acesso às ações e aos serviços [...] (***** $\left.{ }^{*}{ }^{*} j_{1} 102\right)$.

O Cluster 2 - Finalidades da Regulação apresenta os Statements 6, 7, 9, 23, 24 e 25, sendo classificados em três dimensões opostas. Os Statements 6, 7 e 9 consideram que a capacitação do regulador, o treinamento das equipes e a motivação do servidor são fatores importantes que objetivam a melhoria da qualificação da informação e sua consequente transparência, conforme citam os Statements 23 e 25, o que proporcionará um maior controle e monitoramento da regulação do acesso aos serviços de saúde, citado pelo Statement 24. Nota-se uma estreita relação das ideias apesar de estarem distantes visualmente. De fato, há uma lógica entre a capacitação profissional, o que poderá acarretar uma melhoria da informação com uma consequente melhora no controle e monitoramento:

[...] acredito muito na regulação enquanto processo para possibilitar um acesso equânime e universal aos serviços de saúde, no entanto precisa capacitar a equipe, dar condições de trabalho e transformar em um ambiente mais prazeroso [...] $\left({ }^{* * * * *}\right.$ suj_37).

O Cluster 3 - Processo Regulatório apresenta os Statements 16, 17, 18, 20 e 21 aglomerados, porém distantes do Statement 19. Apesar dos participantes entenderem a regulação do acesso aos serviços públicos de saúde como um processo que deva considerar o suporte adequado a ser dado ao paciente (Statement 16), o perfil do paciente (Statement 17), a referência da região de saúde (Statement 18), a vaga ou oferta na rede (Statement 20) e o transporte (Statement 21), o nível de prioridade assistencial (Statement 19) surge como um ponto que se relaciona com todos os demais, tornando-se o foco do processo regulatório. Outra característica deste Cluster é que seu grau de importância foi o mais elevado, demonstrando maior coesão entre as ideias relatadas pelos participantes.

Tal achado assemelha-se aos estudos de Pauly et al. ${ }^{47}$ no Canadá, Kuunders et al. ${ }^{48}$ na Holanda, Cambon et al. ${ }^{49}$ na França e Anderson et al. ${ }^{50}$ nos Estados Unidos que utilizaram o mapeamento conceitual para a análise de sistemas complexos de saúde, fornecendo dados para teorizar contra as desigualdades em saúde nos vários níveis de atenção, levantando estratégias para o aumento da equidade na saúde.

Por fim, o Cluster 4 - Desafios da Regulação, representado pelos Statements 8, 10, 11, 12, 13, 14 e 15 , traduz a percepção dos participantes como o enfrentamento de desafios ou dificuldades a serem vencidas pela regulação como a compreensão do momento oportuno da ação regulatória (Statement 8), a integração das centrais de regulação (Statement 10), a escassez recursos (Statement 11), a melhoria do nível de prioridade assistencial (Statement 13), a qualificação da demanda identificada (Statement 14) e o espaço físico das centrais de regulação (Statement 15). Interessante notar que o Statement 12 se apresenta em uma posição mediana entre os demais pontos, sendo traduzido pela gestão/direção da regulação como a força motriz para a superação dos desafios elencados:

[...] acredito que mesmo com todas as dificuldades que encontramos no nosso dia a dia a regulação é a melhor forma de garantir um acesso aos serviços de saúde mais equânime [...] (***** suj_99).

Assim, considerando o conteúdo das falas dos participantes, a aplicação do mapeamento conceitual pode indicar um conhecimento bem fundamentado sobre os processos de regulação voltadas para o princípio da equidade. Porém, a integração e a interação dos processos de regulação, complexos por natureza, dependerão de ações a serem desenvolvidas pela gestão, tendo como prioridade a capacitação dos profissionais, a melhoria da transparência da informação e a 
adequação do nível de prioridade assistencial para a efetividade de um acesso equânime, racional e oportuno aos usuários do sistema de saúde.

Notadamente, o mapeamento conceitual como recurso metodológico, concentra diferentes perspectivas, conhecimentos e experiências com vistas a encontrar soluções para a resolução de problemas em saúde pública, tornando-se uma ferramenta factível na avaliação de processos colaborativos e participativos, como é o caso da regulação do acesso, mantendo-se o rigor e a credibilidade científica.

\section{Considerações finais}

Busca-se na regulação um meio de se garantir o acesso à saúde, ao utilizar estratégias que possam adequar a relação entre a oferta e a demanda dos serviços, sendo este um dos desafios prementes aos gestores do SUS.

Para tanto, novas ferramentas devem ser pensadas uma vez que a regulação apresenta uma estreita interface com o planejamento, a avaliação, o controle e monitoramento nos diversos níveis de atenção à saúde. Contempla-se aqui o uso da ferramenta de mapeamento conceitual como estratégia de gestão indutora de melhorias dos processos de trabalho até mesmo os mais complexos, como é o caso da regulação em saúde.

Metodologicamente, o uso do mapeamento conceitual neste estudo mostrou-se um instrumento de análise diferenciado, pois contribuiu na identificação de mecanismos causais relacionando-os entre si para melhor compreensão da complexidade do processo regulatório vigente.
Refletiu a lógica do conhecimento embutida nas ideias expressas pelos participantes, assim como, a síntese de visões de diferentes partes interessadas (stakeholders). Colocou em relevo as perspectivas divergentes e convergentes, criando, coletivamente, elementos que orientam a priorização dos processos de trabalho e possíveis melhorias no planejamento e avaliação em um processo de cocriação.

A aplicação do mapeamento conceitual ao processo de regulação do acesso aos serviços públicos de saúde demostra sua potencialidade como uma ferramenta importante na gestão para promover a discussão interdisciplinar, visto que facilita a organização de conceitos e proposições que podem ser trabalhados em projetos complexos na área da saúde, além de clarificar as ações necessárias para se garantir o acesso com equidade e, por conseguinte, a integralidade da atenção à saúde, essenciais à qualificação do sistema de saúde.

Entretanto, demonstra limitações quanto à disponibilidade do método em língua portuguesa, sendo necessárias adaptações que possibilitem o correto emprego das etapas preconizadas. Além disso, a escassez de trabalhos publicados no Brasil que relatem a utilização do mapeamento conceitual para pesquisas em saúde contrasta com a realidade comparada à literatura internacional.

Cabe a reflexão quanto ao tipo de instrumento metodológico a ser adotado como processo de avaliação não só da regulação, mas em todos os serviços de saúde em busca da eficiência e equidade, considerando a acessibilidade, tecnologias e profissionais envolvidos, aspectos estes essenciais à manutenção de qualquer sistema de saúde.

\section{Colaboradores}

Os autores citados participaram da concepção e revisões deste manuscrito aprovando-o e concordando com sua submissão no periódico Ciência \& Saúde Coletiva. 


\section{Referências}

1. Assis MM, Jesus WL. Acesso aos serviços de saúde: abordagens, conceitos, políticas e modelo de análise. Cien Saude Colet 2012; 17(11):2865-2875.

2. McIntyre D, Mooney G, organizadores. The economics of health equity. New York: Cambridge University; 2007.

3. Aday LA, Andersen R. A framework for the study of access to medical care. Health Serv Res 1974; 9(3):208220 .

4. Cechinel C. Regulação em Saúde: um diálogo com o princípio da equidade [dissertação]. Santa Catarina: Universidade Federal de Santa Catarina; 2014.

5. Viacava F, Ugá MAD, Porto S, Laguardia J, Moreira RS. Avaliação de desempenho de sistemas de saúde: um modelo de análise. Cien Saude Colet 2012; 17(4):921934.

6. Starfield B. Acessibilidade e primeiro contato: a "porta”. In: Starfield B, organizador. Atenção primária equilíbrio entre necessidades de saúde, serviços e tecnologia. Brasília: UNESCO/MS; 2002. p. 207-245.

7. Albuquerque MS, Lima LP, Costa AM, Melo Filho DA. Regulação assistencial no Recife: possibilidades e limites na promoção do acesso. Saude Soc 2013; 22(1):223-236.

8. Lima MRM, Silva MVS, Bezerra CJW, Silva LMS, Dourado HHM, Silva AA. Regulação em saúde: conhecimento dos profissionais da estratégia saúde da família. Rev Rene 2013; 14(1):23-31.

9. Gawryszewski ARB, Oliveira DC, Gomes AMT. Acesso ao SUS: representações e práticas de profissionais desenvolvidas nas Centrais de Regulação. Physis 2011; 19:604-609.

10. Scriven E. The future of regulation and governance. JRSH 2007; 127(2):72-77.

11. Jewel T, Wilkinson J. Health and social care regulation in Wales: an integrated system of political, corporate and professional governance for improving public health. JRSH 2008; 128(6):306-312.

12. Vilarins GCM, Shimizu HE, Gutierrez MMU. A regulação em saúde: aspectos conceituais e operacionais. Saude Debate 2012; 36(95):640-647.

13. Crozier M. Estado modesto, Estado moderno. Estratégia para uma outra mudança. Brasília, Funcep; 1989.

14. Brasil. Ministério da Saúde (MS). Secretaria de Atenção à Saúde. Portaria MS/GM no 1.559 , de $1^{\circ}$ de agosto de 2008. Institui a Política Nacional de Regulação do Sistema Único de Saúde. Diário Oficial da União 2008; 4 ago.

15. Brasil. Ministério da Saúde (MS). Controle, regulação e avaliação. Brasília: MS; 2002.

16. Brasil. Ministério da Saúde (MS). Secretaria da Assistência à Saúde. Regulação no setor de saúde: em direção aos seus fundamentos públicos. Brasília: MS; 2004.

17. Brasil. Ministério da Saúde (MS). Secretaria de Atenção à Saúde, Departamento de Regulação, Avaliação e Controle de Sistemas. Diretrizes para a implantação de Complexos Reguladores. Brasília: MS; 2006.

18. Batista SR, Vilarins GCM, Lima MG, Silveira TB. O Complexo Regulador em Saúde do Distrito Federal, Brasil, e o desafio da integração entre os níveis assistenciais. Cien Saude Colet 2019; 24(6):2043-2052.
19. Mendes EV. Os sistemas de serviços de saúde: o que os gestores deveriam saber sobre essas organizações complexas. Fortaleza: Escola de Saúde Pública do Ceará; 2002.

20. Trochim WM: An introduction to concept mapping for planning and evaluation. Eval Program Plann 1989; 12:1-16.

21. Kremer R. Visual Languages for Knowledge Representation. In: Proceedings of KA W'98 Eleventh Workshop on Knowledge Acquisition, Modelling and Management. Canada; 1995. p. 124-129.

22. Kolb DG, Shepherd D. Concept mapping organizational cultures. J Manag Inquiry 1997; 6:282-295.

23. Pammer W, Haney M, Wood BM, Brooks RG, Morse K, Hicks P, Handler EG, Rogers H, Jennett P. Use of Telehealth to extend child protection team services. Pediatrics 2001; 108(3):584-590.

24. Trochim WM, Cook JA, Setze RJ. Using concept mapping to develop a conceptual framework of staff's views of a supported employment program for individuals with severe mental illness. J Consult Clin Psychol 1994; 62(4):766-775.

25. Trochim W, Kane M. Concept mapping: An introduction to structured conceptualization in health care. Int J Qual Health Care 2005; 17(3):187-191.

26. Trochim WM, Cabrera DA, Milstein B, Gallagher RS, Leischow SJ. Practical challenges of systems thinking and modeling in public health. Am. J. Public Health 2006; 96(3):538-546.

27. Kane M, Trochim WM. Concept mapping for planning and evaluation. Thousand Oaks: Sage Publications; 2007.

28. Donnelly JP. A systematic review of concept mapping dissertations. Eval Program Plann 2017; 60:186-193.

29. Anderson LA, Slonim A. Perspectives on the strategic uses of concept mapping to address public health challenges. Eval Program Plann 2017; 60:194-201.

30. Goicolea I, Carson D, San Sebastian M, Christianson M, Wiklund M, Hurtig AK. Health care access for rural youth on equal terms? A mixed methods study protocol in northern Sweden. Int J Equity Health 2018; 17(1):6.

31. Bradbury-Jones C, Breckenridge JP, Devaney J, Duncan F, Kroll T, Lazenbatt A, Taylor J. Priorities and strategies for improving disabled women's access to maternity services when they are affected by domestic abuse: a multi-method study using concept maps. BMC Pregnancy Childbirth 2015; 15:350.

32. Johnson DB, Quinn E, Sitaker M, Ammerman A, Byker C, Dean W, Fleischhacker S, Kolodinsky J, Pinard C, Pitts SBJ, Sharkey J. Developing an agenda for research about policies to improve access to healthy foods in rural communities: a concept mapping study. BMC Public Health 2014; 14(1):592.

33. Willging CE, Sommerfeld DH, Jaramillo ET, Lujan E, Bly RS, Debenport EK, Verney SP, Lujan R. Improving native American elder access to and use of health care through effective health system navigation. $\mathrm{BMC} \mathrm{He}$ alth Serv Res 2018; 18(1):464.

34. Ahmad F, Mahmood S, Pietkiewicz I, McDonald L, Ginsburg O. Concept mapping with South Asian immigrant women: barriers to mammography and solutions. J Immigr Minor Health 2012; 14(2):242-250. 
35. Vives-Cases C, Goicolea I, Hernández A, Sanz-Barbero B, Davó-Blanes MC, La Parra-Casado D. Priorities and strategies for improving Roma women's access to primary health care services in cases on intimate partner violence: A concept mapping study. Int J Equity Health 2017; 16(1):96.

36. Novak JD, Gowin DB. Learning how to learn. Boston: Cambridge University Press: 1984.

37. Ruiz-Moreno L, Sonzogno MC, Batista SHS, Batista NA. Mapa conceitual: ensaiando critérios de análise. Cien Educ (Bauru) 2007; 13(3):453-463.

38. Lins MPE, Antoun Netto SO, Lobo MSC. Multimethodology applied to the evaluation of Healthcare in Brazilian municipalities. Health Care Manag Sci 2019; 22(2):197-214.

39. Figueiredo TA, Hartz ZMA. Avaliação de desempenho da regulação em saúde. An Inst Hig Med Trop 2017; 16(Supl. 3):S19-S28.

40. Bussab W, Bolfarine H. Elementos de amostragem. São Paulo: Edgar Blucher; 2005.

41. Camargo BV, Justo AM. Tutorial para uso do software de análise textual IRAMUTEQ [Internet]. UFSC; 2013 [acessado 2019 out 5]. Disponível em: http:// www.iramuteq.org/documentation/fichiers/tutoriel -en-portugais.

42. Camargo BV, Justo AM. IRaMuTeQ: um software gratuito para análise de dados textuais. Temas Psicol 2013 21(2):513-518.

43. Salvador PTCO, Gomes ATL, Rodrigues CCFM, Chiavone FBT, Alves KYA, Bezerril MS, Santos VEP. O uso do software IRaMuTeQ nas pesquisas brasileiras da área da saúde: uma scoping review. Rev Bras Promo Saude 2018; 31(Supl.):1-9.

44. Souza MAR, Wall ML, Thuler ACMC, Lowen IMV, Peres AM. The use of IRaMuTeQ software for data analysis in qualitative research. Rev Esc Enferm USP 2018; 52:1-7.

45. Burke JG, O'Campo P, Peak GL, Gielen AC, McDonnell KA, Trochim WM. An introduction to concept mapping as a participatory public health research methodology. Qual Health Res 2005; 15(10):1392-1410.
46. Smallman M. Public Understanding of Science in turbulent times III: Deficit to dialogue, champions to critics. Public Underst Sci 2016; 25(2):186-197.

47. Pauly B, MacDonald M, Hancock T, Martin W, Perkin $K$. Reducing health inequities: the contribution of core public health services in BC. BMC Public Health 2013; 13:550.

48. Kuunders T, van Bon-Martens M, van de Goor I, Paulussen $\mathrm{T}$, van Oers $\mathrm{H}$. Towards local implementation of Dutch health policy guidelines: A concept-mapping approach. Health Promot Int 2017; 33(4):635-647.

49. Cambon L, Minary L, Ridde V, Alla FA. Tool to analyze the transferability of health promotion interventions. BMC Public Health 2013; 13:1184.

50. Anderson LA, Day KL, Vandenberg AE. Using a concept map as a tool for strategic planning: The Healthy Brain Initiative. Prev Chronic Dis 2011; 8(5):A117.

Artigo apresentado em 12/12/2019

Aprovado em 04/10/2020

Versão final apresentada em 06/10/2020

Editores-chefes: Romeu Gomes, Antônio Augusto Moura da Silva 